\title{
The $k$-independent graph of a graph
}

\author{
Davood Fatehi $^{a}$, Saeid Alikhani ${ }^{a, *}$, Abdul Jalil M. Khalaf ${ }^{b}$ \\ January 3, 2020 \\ ${ }^{a}$ Department of Mathematics, Yazd University, 89195-741, Yazd, Iran \\ davidfatehi@yahoo.com, alikhani@yazd.ac.ir \\ ${ }^{b}$ Department of Mathematics, Faculty of Computer Science and Mathematics \\ University of Kufa, PO Box 21, Najaf, Iraq \\ abduljaleel.khalaf@uokufa.edu.iq
}

\begin{abstract}
Let $G=(V, E)$ be a simple graph. A set $I \subseteq V$ is an independent set, if no two of its members are adjacent in $G$. The $k$-independent graph of $G, I_{k}(G)$, is defined to be the graph whose vertices correspond to the independent sets of $G$ that have cardinality at most $k$. Two vertices in $I_{k}(G)$ are adjacent if and only if the corresponding independent sets of $G$ differ by either adding or deleting a single vertex. In this paper, we obtain some properties of $I_{k}(G)$ and compute it for some graphs.
\end{abstract}

Keywords: independence number; $k$-independent graph; reconfiguration.

AMS Subj. Class.: 05C60, 05C69

\section{Introduction}

Given a simple graph $G=(V, E)$, a set $I \subseteq V$ is an independent set of $G$, if there is no edge of $G$ between any two vertices of $I$. A maximal independent set is an independent set that is not a proper subset of any other independent set. A maximum independent set is an independent set of greatest cardinality for $G$. This cardinality is called independence number of $G$, and is denoted by $\alpha(G)$. Reconfiguration problems have been studied often in recent years. These

\footnotetext{
${ }^{*}$ Corresponding author
} 
arise in settings where the goal is to transform feasible solutions to a problem in a step-by-step manner, while maintaining a feasible solution throughout.

For the study of dominating set reconfiguration problem: given two dominating sets $S$ and $T$ of a graph $G$, both of size at most $k$, is it possible to transform $S$ into $T$ by adding and removing vertices one-by-one, while maintaining a dominating set of size at most $k$ throughout? Regarding to this dominating set reconfiguration problem, recently the $k$-dominating graph of a graph $G$ has defined in [9]. The $k$-dominating graph of $G, D_{k}(G)$, is defined to be the graph whose vertices correspond to the dominating sets of $G$ that have cardinality at most $k$. Two vertices in $D_{k}(G)$ are adjacent if and only if the corresponding dominating sets of $G$ differ by either adding or deleting a single vertex. Authors in [9], gave conditions that ensure $D_{k}(G)$ is connected. In [1] authors proved that if $G$ is a graph without isolated vertices of order $n \geq 2$ and with $G \cong D_{k}(G)$, then $k=2$ and $G=K_{1, n-1}$ for some $n \geq 4$. It is also proved that for a given $r$ there exist only a finite number of $r$-regular, connected dominating graphs of connected graphs $([1])$.

One of the most well-studied problem in reconfiguration problems, is the reconfiguration of independent sets. For a graph $G$ and integer $k$, the independent sets of size at least/exactly $k$ of $G$ form the feasible solutions. Independent sets are also called token configurations, where the independent set vertices are viewed as tokens [4]. Deciding for existence of a reconfiguration between two $k$-independent sets with at most $\ell$ operations is strongly NP-complete ([10]). Bonamy and Bousquet in [3] have considered the $k$-TAR reconfiguration graph, $T A R_{k}(G)$, as follows:

A $k$-independent set of $G$ is a set $S \subseteq V$ with $|S| \geq k$, such that no two elements of $S$ are adjacent. Two $k$-independent sets $I$ and $J$ are adjacent if they differ on exactly one vertex. This model is called the Token Addition and Removal (TAR). Authors in [3] provided a cubic-time algorithm to decide whether $T A R_{k}(G)$ is connected when $G$ is a graph which does not contain induced paths of length 4. Their work solves an open question in [4]. Also they described a linear-time algorithm which decides whether two elements of $T A R_{k}(G)$ are in the same connected component. As usual we denote the complete graph, path and cycle of order $n$ by $K_{n}, P_{n}$ and $C_{n}$, respectively. Also $K_{1, n}$ is the star graph with $n+1$ vertices.

In the next section, we study the $k$-independent graph of a graph $G$. In Section 3, we study the $\alpha$-independent graph of a graph. Finally in Section 3, we exclude the empty set from the family set of independent sets of $G$, denote the new $k$-independent graph of $G$ by $I_{k}^{*}(G)$ and study its connectedness. 


\section{The $k$-independent graph of a graph}

In this section we shall study the $k$-independent graph of a graph $G$. First let to rewrite the definition of the reconfiguration graph $T A R_{k}(G)$, as follows. For a graph $G$ and a non-negative integer $k$, the $k$-independent graph of $G, I_{k}(G)$, is defined to be the graph whose vertices correspond to the independent sets of $G$ that have cardinality at most $k$. Two vertices in $I_{k}(G)$ are adjacent if and only if the corresponding independent sets of $G$ differ by either adding or deleting a single vertex. As an example, Figure 1 shows $I_{3}\left(K_{1,3}\right)$.
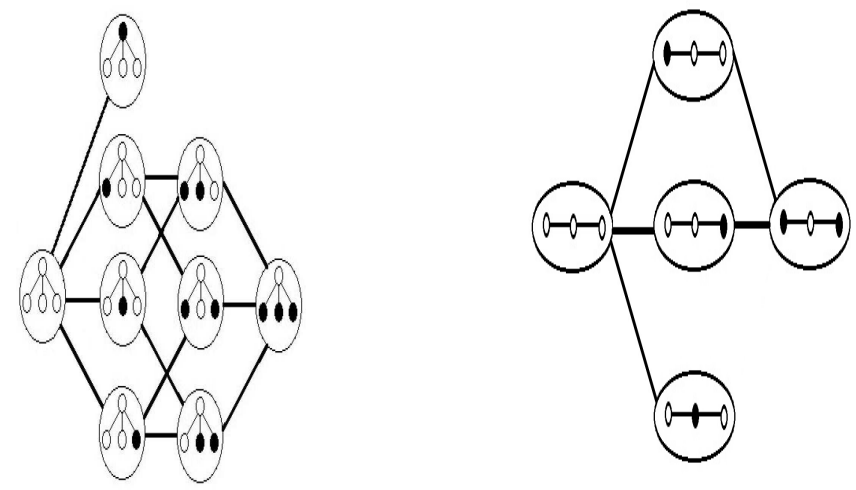

Figure 1: Graphs $I_{3}\left(K_{1,3}\right)$ and $I_{2}\left(P_{3}\right)$, respectively.

Note that $k$-dominating and $k$-independent graph are similar to recent work in graph colouring, too. Given a graph $H$ and a positive integer $k$, the $k$-colouring graph of $H$, denoted $G_{k}(H)$, has vertices corresponding to the (proper) $k$-vertexcolourings of $H$. Two vertices in $G_{k}(H)$ are adjacent if and only if the corresponding vertex colourings of $G$ differ on precisely one vertex. Authors in [5, 6, 7, 8] studied the connectedness of $k$-colouring graphs. Also they studied their hamiltonicity. Let to introduce a notation. Let $A$ and $B$ be independent sets of $G$ of cardinality at most $k$. We use the notation $A \leftrightarrow B$, if there is a path in $I_{k}(G)$ joining $A$ and $B$. It is easy to see that for every $A, B \in I_{k}(G), A \leftrightarrow B$ if and only if $B \leftrightarrow A$ and if $A \supseteq B$, then $A \leftrightarrow B$ and $B \leftrightarrow A$.

The following theorem, gives some properties of the $k$-independent graph of a graph:

Theorem $2.1 \quad$ (i) If $G$ is a graph of order $n$, then $I_{1}(G) \cong K_{1, n}$.

(ii) For every graph $G$ and every $0 \leq k \leq \alpha(G)$, the independent graph $I_{k}(G)$ is connected and $\Delta\left(I_{k}(G)\right)=|V(G)|$.

(iii) For every graph $G$, the independent graph $I_{k}(G)$ is a bipartite graph. 
(iv) If $G \not \overline{K_{n}}$, then $I_{k}(G)$ is not a regular graph.

(v) If $G ¥ \overline{K_{n}}$ then $I_{k}(G)$ is not a vertex-transitive graph, and so is not a Cayley graph.

\section{Proof.}

(i) It follows from the definition.

(ii) It is straightforward.

(iii) Let $X$ be the set of independent sets of size less than $k+1$ of $G$ with odd cardinality and $Y$ be the set of independent sets of size less than $k+1$ with even cardinality. It is clear that $X \cup Y=V\left(I_{k}(G)\right)$ and $X \cap Y=\phi$. Suppose that $A, B \in X$, then $(A \backslash B) \cup(B \backslash A)$ cannot be a vertex of $I_{k}(G)$. Because $|A|=|B|$ or ||$A|-| B|| \geq 2$. So $A B$ is not an edge of $I_{k}(G)$ and with similar argument we have this for two vertices in $Y$. Therefore $I_{k}(G)$ is a bipartite graph with parts $X$ and $Y$.

(iv) Let $G$ be a graph of order $n$. The empty set is an independent set of $G$ which has degree $n$ in $I_{k}(G)$. Let $I_{1}$ be an independent set of $G$ with $\left|I_{1}\right|=\alpha(G)$. We know that $I_{1}$ is adjacent to $\alpha$ independent sets. Since $G \varsubsetneqq \overline{K_{n}}$, we have $\alpha(G) \neq n$. Therefore $I_{k}(G)$ is not a regular graph.

(v) It follows from Part (iv).

Theorem $2.2 \quad$ (i) Let $G$ be a graph of order $n$. There is no integer $k$, such that $I_{k}(G) \cong G$.

(ii) If $G ¥ K_{n}$, then the girth of $I_{k}(G)$ is 4 .

(iii) Let $G \neq K_{n}$ be a graph. Then for all integers $k \geq 2, I_{k}(G)$ is not a tree.

\section{Proof.}

(i) Since for every integer number $k \geq 1,\left|V\left(I_{k}(G)\right)\right| \geq n+1$, so we have the result.

(ii) Let $v_{1}$ and $v_{2}$ be two non-adjacent vertices of graph $G$. So $\left\{v_{1}\right\}$ and $\left\{v_{2}\right\}$ are two independent sets of $G$ and therefore two vertices of $I_{k}(G)$. Now $\emptyset$, $\left\{v_{1}\right\},\left\{v_{1}, v_{2}\right\},\left\{v_{2}\right\}, \emptyset$ is a cycle in $I_{k}(G)$ and this is the shortest cycle in $I_{k}(G)$. Therefore the girth of $I_{k}(G)$ is 4 .

(iii) It follows from Part $(i i)$. 


\section{The $\alpha$-independent graph of some graphs}

Let $G$ be a simple graph with independence number $\alpha$. Looks that in the among of $k$-independent graph of $G$, the $\alpha$-independent graph of $G$ is more important. In this section, we study the $\alpha$-independent graph of some graphs. To study the $\alpha$-independent graph of $G$, we are interested to know the order of $I_{\alpha}(G)$. Let $i_{k}$ be the number of independent sets of cardinality $k$ in $G$. The polynomial

$$
I(G, x)=\sum_{k=0}^{\alpha(G)} i_{k} x^{k},
$$

is called the independence polynomial of $G([2])$. Obviously $I(G, 1)$ gives the number of all independent sets of a graph $G$. In other words, $\left|V\left(I_{\alpha}(G)\right)\right|=$ $I(G, 1)$. Since $I\left(K_{n}, x\right)=1+n x$, we have $I\left(K_{n}, 1\right)=n+1$. Therefore we have the following easy result:

Theorem 3.1 For any integer $k>1$, there is some connected graph $G$ such that $\left|V\left(I_{\alpha}(G)\right)\right|=k$.

The following theorem is about the $\alpha$-independent graph of stars:

Theorem 3.2 (i) The $n$-independent graph of $K_{1, n}$, i.e., $I_{n}\left(K_{1, n}\right)$ is a bipartite graph with parts $X$ and $Y$, with $|X|=2^{n-1}$ and $|Y|=2^{n-1}+1$.

(ii) The $n$-independent graph $I_{n}\left(K_{1, n}\right)$ is not Hamiltonian.

\section{Proof.}

(i) Let $X$ be the set of independent sets of $K_{1, n}$ with even cardinality and $Y$ be the set of independent sets of odd cardinality. By Theorem 2.1(iii), $I_{n}\left(K_{1, n}\right)$ is a bipartite graph with parts $X$ and $Y$. Obviously $|X|=\sum_{k=0}^{\left\lfloor\frac{n}{2}\right\rfloor}\left(\begin{array}{c}n \\ 2 k\end{array}\right)$ and since the number of independent sets of $K_{1, n}$ is $I\left(K_{1, n}, 1\right)=2^{n}+1$, we have $|Y|=1+\sum_{k=1}^{\left\lfloor\frac{n}{2}\right\rfloor}\left(\begin{array}{c}n \\ 2 k-1\end{array}\right)$. Therefore we have the result.

(ii) Since a bipartite graph with different number of vertices in its parts is not a Hamiltonian graph, so the $n$-independent graph $I_{n}\left(K_{1, n}\right)$ is not a Hamiltonian graph.

Here we consider the $\alpha$-independent of some another graphs. Figure 1 shows the $I_{2}\left(P_{3}\right)$.

Theorem 3.3 For every $n \in \mathbb{N}, \delta\left(I_{\alpha}\left(P_{n}\right)\right)=\left\lfloor\frac{n}{2}\right\rfloor$. 
Proof. The minimum degree of vertices of $I_{\left\lceil\frac{n}{2}\right\rceil}\left(P_{n}\right)$ is due to maximal independent sets of $P_{n}$ with minimum cardinality. These vertices are adjacent to $n-\left\lceil\frac{n}{2}\right\rceil=\left\lfloor\frac{n}{2}\right\rfloor$ of independent sets with less cardinality.

Here we shall obtain information on the Hamiltonicity of $\alpha$-independent of some specific graphs. Using the value of the independence polynomial at -1 , we have $I(G ;-1)=i_{0}-i_{1}+i_{2}-\ldots+(-1)^{\alpha} i_{\alpha}=f_{0}(G)-f_{1}(G)$, where $f_{0}(G)=$ $i_{0}+i_{2}+i_{4}+\ldots, f_{1}(G)=i_{1}+i_{3}+i_{5}+\ldots$ are equal to the numbers of independent sets of even size and odd size of $G$, respectively. $I(G,-1)$ is known as the alternating number of independent sets. We need the following theorem:

Theorem 3.4 [11] For $n \geq 1$, the following hold:

(i) $I\left(P_{3 n-2} ;-1\right)=0$ and $I\left(P_{3 n-1} ;-1\right)=I\left(P_{3 n} ;-1\right)=(-1)^{n}$;

(ii) $I\left(C_{3 n} ;-1\right)=2(-1)^{n}, I\left(C_{3 n+1} ;-1\right)=(-1)^{n}$ and $I\left(C_{3 n+2} ;-1\right)=(-1)^{n+1}$;

(iii) $I\left(W_{3 n+1} ;-1\right)=2(-1)^{n}-1$ and $I\left(W_{3 n} ;-1\right)=I\left(W_{3 n+2} ;-1\right)=(-1)^{n}-1$.

Corollary 3.5 For all positive integer $n$, the graphs $I_{\alpha}\left(P_{3 n-1}\right), I_{\alpha}\left(P_{3 n}\right), I_{\alpha}\left(C_{n}\right)$ and $I_{\alpha}\left(W_{n}\right)$ are not Hamiltonian.

Proof. We know that $I_{\alpha}\left(P_{n}\right), I_{\alpha}\left(C_{n}\right)$ and $I_{\alpha}\left(W_{n}\right)$ are bipartite graphs with parts containing the independent sets of even and odd cardinality. By Theorem 3.4. theses bipartite graphs have parts with different cardinality. Therefore we have the result.

\section{Connectedness of $I_{k}^{*}(G)$}

As we have seen in the Section 2, since the empty set is an independent set of any graph, so the $k$-independent graph $I_{k}(G)$ is a connected graph. Let us to do not consider empty set in the study of $k$-independent graph.

Suppose that $\mathcal{I}$ is a family of all independent sets of graph $G$. If we put $V\left(I_{k}(G)\right)=\mathcal{I} \backslash \emptyset$, then we denote the $k$-independent graph of $G$, by $I_{k}^{*}(G)$. Note that in this case, for some $k$ and $G, I_{k}^{*}(G)$ is disconnected and for some $k$ and $G$ is connected. For example, the Figure 2 shows $I_{3}^{*}\left(K_{1,3}\right)$ and $I_{2}^{*}\left(C_{4}\right)$, which are disconnected graphs with two components. Also Figure 3 shows $I_{2}^{*}\left(W_{5}\right)$ and $I_{3}^{*}\left(P_{5}\right)$, respectively. Observe that $I_{3}^{*}\left(P_{5}\right)$ is connected and $I_{2}^{*}\left(W_{5}\right)$ is disconnected with three components. Theorem 2.2 implies that for any graph $G \neq K_{n}$, and for all integers $k \geq 2, I_{k}(G)$ is not a tree, but as we see in the Figure 3, the graph $I_{k}^{*}(G)$ can be a forest. This naturally raises the question: For which graph $G$, the component of $I_{k}^{*}(G)$ is a forest? What is the number of components?

The following theorem is a sufficient condition for disconnectedness of $I_{\alpha}^{*}(G)$. 

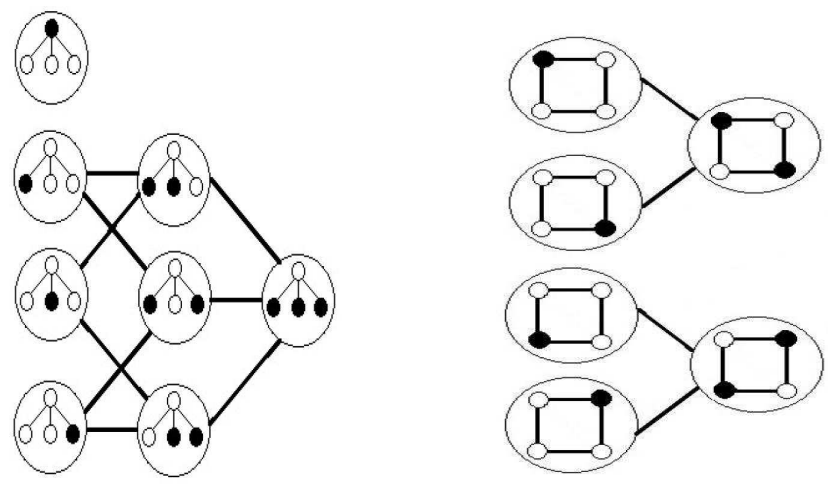

Figure 2: Graphs $I_{3}^{*}\left(K_{1,3}\right)$ and $I_{2}^{*}\left(C_{4}\right)$, respectively.

Theorem 4.1 If a graph $G$ of order $n$ has a vertex of degree $n-1$, then $I_{\alpha}^{*}(G)$ is disconnected.

Proof. Let $v$ be a vertex of degree $n-1$. Obviously $\{v\}$ is a non-empty independent set of $G$, and so is an isolated vertex of $I_{\alpha}^{*}(G)$.

Note that the converse of Theorem 4.1 is not true. For example $I_{2}^{*}\left(C_{4}\right)$ has two components, but $C_{4}$ is 2-regular (Figure 3). Now, we state the following theorem:

Theorem 4.2 Let $K_{n_{1}, n_{2}, \ldots, n_{m}}$ be a complete m-partite graph, then $I_{\alpha}^{*}\left(K_{n_{1}, n_{2}, \ldots, n_{m}}\right)$ has $m$ connected components.

Proof. Let $X_{1}$ and $X_{2}$ be two arbitrary parts of $K_{n_{1}, n_{2}, \ldots, n_{m}}$. Suppose that $I_{1}$ contains all nonempty subsets of part $X_{1}$ and $I_{2}$ contains all nonempty sets of part $X_{2}$. Obviously, each member of $I_{1}$ and each member of $I_{2}$ are independent sets of $K_{n_{1}, n_{2}, \ldots, n_{m}}$ and so they are vertices of $I_{\alpha}^{*}\left(K_{n_{1}, n_{2}, \ldots, n_{m}}\right)$. No member of $I_{1}$ is adjacent to a member of $I_{2}$ in $I_{\alpha}^{*}\left(K_{n_{1}, n_{2}, \ldots, n_{m}}\right)$. So $I_{\alpha}^{*}\left(K_{n_{1}, n_{2}, \ldots, n_{m}}\right)$ is a disconnected graph. Since the members of $I_{1}$ (and the members of $I_{2}$ ) form a connected graph, therefore we have $m$ components.

It is obvious that, for all graph $G$ with $\alpha(G)=2, I_{2}^{*}(G)$ is a forest.

Theorem 4.3 For all graph $G$ with $\alpha(G)>2$, the components of $I_{k}^{*}(G), 2 \leq$ $k \leq \alpha$, are not forest.

Proof. We consider two following cases:

Case 1. If $k=2$. Let $\left\{v_{1}, v_{2}, v_{3}\right\}$ be an independent set of $G$. So $\left\{v_{1}\right\},\left\{v_{2}\right\}$, $\left\{v_{3}\right\},\left\{v_{1}, v_{2}\right\},\left\{v_{1}, v_{3}\right\}$ and $\left\{v_{2}, v_{3}\right\}$ are independent sets of $G$ and vertices of 
$I_{k}^{*}(G)$. Therefore $\left\{v_{1}\right\},\left\{v_{1}, v_{2}\right\},\left\{v_{2}\right\},\left\{v_{2}, v_{3}\right\},\left\{v_{3}\right\},\left\{v_{1}, v_{3}\right\},\left\{v_{1}\right\}$ make a cycle in $I_{k}^{*}(G)$.

Case 2. If $k>2$. Let $\left\{v_{1}, v_{2}, v_{3}\right\}$ be an independent set of $G$. So $\left\{v_{1}\right\},\left\{v_{1}, v_{2}\right\}$ and $\left\{v_{1}, v_{3}\right\}$ are independent sets of $G$ and vertices of $I_{k}^{*}(G)$. Therefore $\left\{v_{1}\right\}$, $\left\{v_{1}, v_{2}\right\},\left\{v_{1}, v_{2}, v_{3}\right\},\left\{v_{1}, v_{3}\right\},\left\{v_{1}\right\}$ make a cycle in $I_{k}^{*}(G)$ and so $I_{k}^{*}(G)$ is not a forest.

Note that if $G$ is a graph of order $n$ with $\alpha(G)>2$, then similar to Theorem 4.3, $I_{k}^{*}(G)$ cannot be a path, cycle and a chordal graph.
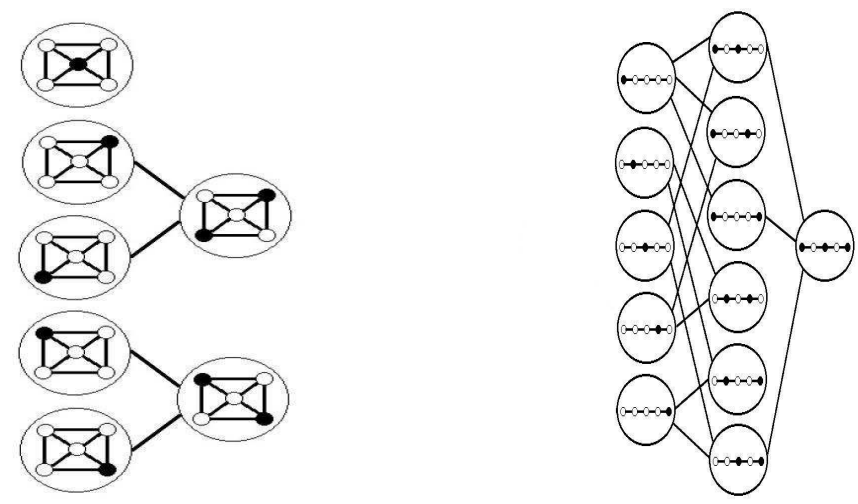

Figure 3: Graphs $I_{2}^{*}\left(W_{5}\right)$ and $I_{3}^{*}\left(P_{5}\right)$, respectively.

Theorem 4.4 Let $G$ be a (non complete) bipartite graph of order $n>4$. Then $I_{k}^{*}(G)$ is connected.

Proof. Let $I_{1}$ and $I_{2}$ be two independent sets of $G$ and $\left|I_{1}\right|,\left|I_{2}\right| \leq k$, so $I_{1}$ and $I_{2}$ are two vertices of $I_{k}(G)$. If $I_{1} \cap I_{2} \neq \phi$ then $I_{1} \leftrightarrow I_{1} \cap I_{2} \leftrightarrow I_{2}$. If $I_{1} \cap I_{2}=\phi$, we consider two following cases:

Case 1. There are $v_{1} \in I_{1}$ and $v_{2} \in I_{2}$ such that $v_{1}$ and $v_{2}$ are not adjacent then $I_{1} \leftrightarrow\left\{v_{1}\right\} \leftrightarrow\left\{v_{1}, v_{2}\right\} \leftrightarrow\left\{v_{2}\right\} \leftrightarrow I_{2}$.

Case 2. For all $v_{1} \in I_{1}$ and $v_{2} \in I_{2}, v_{1}$ is adjacent to $v_{2}$. So $I_{1} \subset A$ and $I_{2} \subset B$, where $A$ and $B$ are two parts of $G$. Since $G$ is not complete bipartite graph so $I_{1} \neq A$ and $I_{2} \neq B$ and there are $v_{3} \in A$ and $v_{4} \in B$ such that $v_{3} \notin I_{1}$ and $v_{3}$ is not adjacent to $v_{4}$. We put $I_{3}=\left(I_{1} \backslash\left\{v_{1}\right\}\right) \cup\left\{v_{3}\right\}$. So $\left|I_{3}\right|=\left|I_{1}\right|$ and $I_{1} \leftrightarrow I_{1} \backslash\left\{v_{1}\right\} \leftrightarrow I_{3}$ and $I_{3} \leftrightarrow\left\{v_{3}\right\} \leftrightarrow\left\{v_{3}, v_{4}\right\} \leftrightarrow\left\{v_{4}\right\} \leftrightarrow I_{2}$. Therefore $I_{1} \leftrightarrow I_{2}$.

\section{References}

[1] S. Alikhani, D. Fatehi, S. Klavźar, On the structure of dominating graphs, submitted. Available at http://arxiv.org/abs/1512.07514. 
[2] S. Alikhani and Y.H. Peng, Independence roots and independence fractals of certain graphs, J. Appl. Math. Comput., 36 (2011), pp. 89-100.

[3] M. Bonamy and N. Bousquet, Reconfiguring independent sets in cographs, Arxiv preprint, available at http://arxiv.org/abs/1406.1433.

[4] P. Bonsma, Independent set reconfiguration in cographs, Arxiv preprint, available at http://arxiv.org/abs/1402.1587v1.

[5] L. Cereceda, J. van den Heuvel, M. Johnson, Connectedness of the graph of vertex-colourings. Discrete Math. 308, (2009) 913-919.

[6] L. Cereceda, J. van den Heuvel, M. Johnson, Finding paths between 3colorings. J. Graph Theory 67, (2011) 69-82.

[7] K. Choo, G. MacGillivray, Gray code numbers for graphs, Ars Math. Contemp. 4, (2011) 125-139.

[8] R. Haas, The canonical coloring graph of trees and cycles, Ars Math. Contemp. 5 (2012), 149-157.

[9] R. Haas, K. Seyffarth, The $k$-dominating graph, Graphs Combin., 30 (2014) 609-617.

[10] M. Kamiński, P. Medvedev, and M. Milanič, Complexity of independent set reconfigurability problems, Theoretical Computer Science, 439(0) (2012) $9-15$.

[11] V.E. Levit and E. Mandrescu, The independence polynomial of a graph at -1, Arxiv preprint, available at http://arxiv.org/abs/0904.4819. 